\title{
Distributed Object-Oriented Wargame Simulation on Access Grid ${ }^{\star}$
}

\author{
Joong-Ho Lim, Tae-Dong Lee, and Chang-Sung Jeong ${ }^{\star \star}$ \\ School of Electrical Engineering in Korea University, \\ 1-5ka, Anam-Dong, Sungbuk-Ku, Seoul 136-701, Korea \\ $\{\mathrm{jhlim}$, lyadlove\}@snoopy.korea.ac.kr \\ csjeong@charlie.korea.ac.kr
}

\begin{abstract}
This paper presents the design and implementation of Distributed Object-oriented Wargame Simulation(DOWS) on Access Grid(AG). DOWS is an object-oriented distributed simulation system based on a director-actor model. DOWS on AG supports a collaborative environment by providing a virtual venue with high quality audio and real-time video interactive interface for remote users, and allows a groups of users in remote sites to easily participate in the whole simulation. We design an efficient communication scheme between application and AG so that DOWS can be incorporated on AG for collaboration purpose.
\end{abstract}

\section{Introduction}

The simulation can be adapted in many fields such as construction, traffic, measurement and so on. One of them is a war game simulation in military field. Although there are few battles in the world, the military should continue to train their army to improve the operation skill of battle. However, the government must spend expensive budgets to check their strategies and maintain their battle ability, because the real training operation of the army must require high cost and time. Therefore, war-game simulation is regarded as one of the most effective ways to test new strategies and the operation capability of army's force.

However, it requires a large volume of multimedia data as well as many complex processes, and there exists some limit in number of operators and observers due to the space limitation. Also, it is difficult to have video/audio services in collaborative environment. In this paper, we present a Distributed Object-oriented Wargame Simulation(DOWS) system which provides not only distributed simulation environment but also collaborative environment using Access Grid(AG). We shall describe an efficient communication scheme which integrates application into AG so that DOWS can be easily incorporated onto AG for collaborative purpose.

\footnotetext{
* This work has been supported by KIPA-Information Technology Research Center, University research program by Ministry of Information \& Communication, and Brain Korea 21 projects in 2005.

** Corresponding author.
} 
In section 2, we briefly describe the Access Grid, and in section 3, briefly explain DOWS. In section 4, we present an architecture for DOWS on AG. In section 5 , we conclude with future works.

\section{Access Grid}

Grid computing is a specialized form of distributing computing where the application is distributed over a wide-area network and computing nodes are geographically far-off from each other [2]. It comprises Computational, Data and Access Grid. Computational Grid can share computing resources at remote site for high performance computing. Data Grid provides an integrated view for the distributed data. Access Grid(AG), developed by Argonne national laboratory, provides various services such as video/audio conferencing, data sharing, shared application, text-based communication and certificates management for collaboration[3]. It enables remote users to interact each other, and exchange information through video and audio services.

AGTk(Access Grid Toolkit) has been developed using Grid technology 1, and provides all the services offered in Grid. It is composed of AG Server, AG Client and virtual venues. An AG client joins into a virtual venue to collaborate with other members in the venue, and interact through AG server.

\section{Dows System}

DOWS is an object-oriented simulation system based on a director-actor model which can be mapped efficiently on object-oriented and distributed simulation. Director-actor model consists of actors and directors which interact with others. Actor represents a simulation entity or a sub-model in the simulation, and director is a participant in the simulation which controls the actors. Each director generates commands or events to its associated actors which in turn activate the actual simulations by interacting with other actors in the same or different subgroups. A set of actors may be designated as actor group so that each director can issue commands to each member in the actor group simultaneously. In director-actor model, simulation is carried out by actors interacting with each other. Each actor can represent a simulation entity participating in one simulation model or a sub-model in the whole simulation. DOWS consists of four major components which interact with each other on a distributed environment: director, actor, agent and coordinator. First, directors instantiate the simulation model and participate in the simulation concurrently through the interactive communication with the agents which are in charge of the parallel simulation of its associated sub-models using several actors. Second, each actor corresponds to a simulation entity of the simulation model or a sub-model which is a part of the whole simulation model. Several actors can be executed either in one processor or several processors interconnected through a network on a distributed environment. Each actor sends an event message to the channel of the other actor, and maintains the synchronization. Third, the agent provides an efficient virtual real 
time simulation environment which integrates the coordination among directors and actors by supporting time synchronization, simulation message transfer, and network fault detection. each agent can play a role of a message router, and coordinates each other to forward messages between actors and directors. Last, the coordinator runs the whole simulation by sending start message to each actor, or suspends it by sending a block signal to the agents whose input thread in turn notifies its corresponding actors to block.

\section{DOWS on Access Grid}

AG supports collaborative applications through the channel sharing. We classify users into operator and observer: The former can control simulation by issuing commands to the actors directly, and the latter only view the simulation controlled by the former. After login, DOWS operator and observer applications can be started by operators and observers respectively. Figure 1(a) show Venue client and server. Venue server supports various services such as venue creation, synchronization, authorization, registration, and data broadcasting. As in figure 1(b)(c), Venue client creates a relay station for each DOWS operator application, which interacts with other observer applications through its own relay station. Relay stations has a role which relays messages between DOWS application and

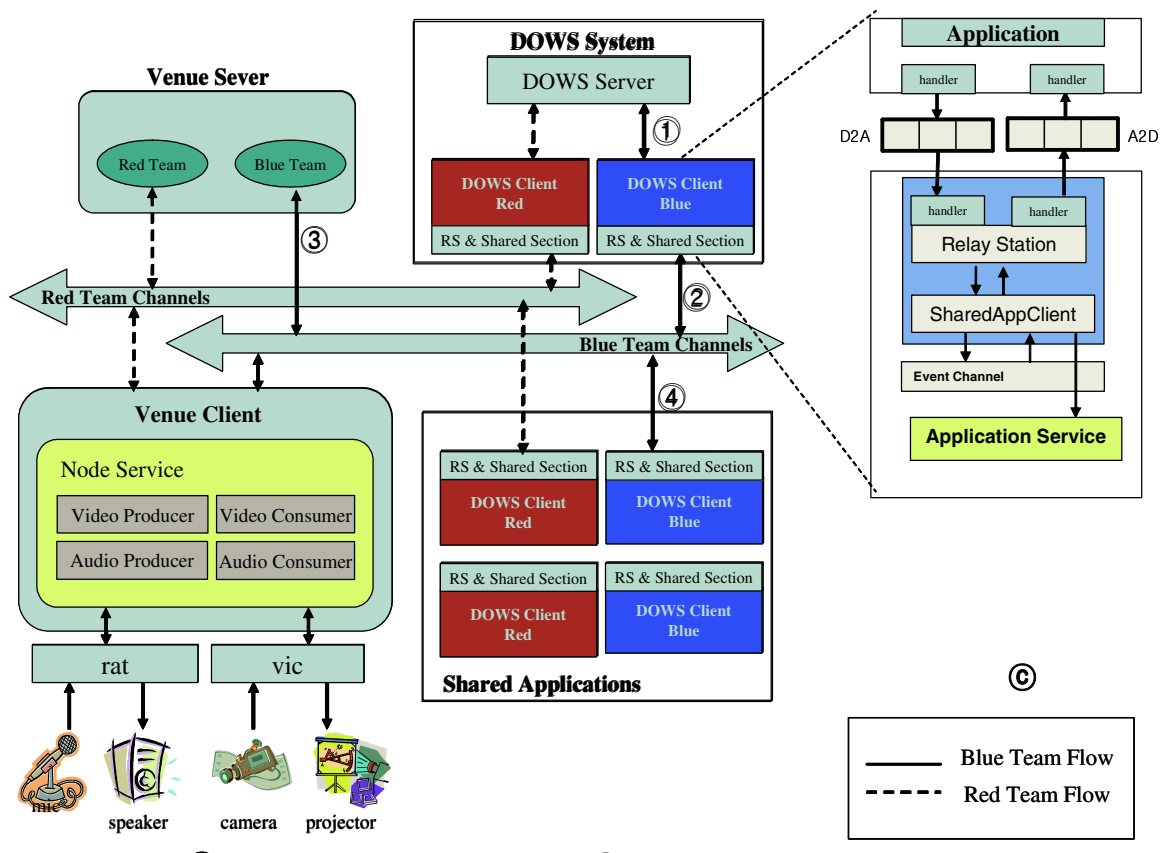

(a)

(D)

Fig. 1. An architecture of DOWS on AG 
AG, and use two message queues between them. Each relay station receives the updated data in the message queue sent from its corresponding DOWS operator application using its message handler, and propagates them to other relay stations in the same venue using the event channel provided by application service in AGTk. Then, all the observer application in the same venue receives the updated data from the message queue sent by the relay station. Application service APIs provided by AGTk support a mechanism for event channel, discovery, coherence, and synchronization among relay stations. Relay station is implemented using python script language, and makes use of the functions in the SharedAppClient class which exploits application service APIs provided by AGTk.

For example, as shown in figure 1(a), two venues called "Blue Team Venue" and "Red Team Venue" are created in the Venue server. Suppose DOWs server, Venue clients and server exist already. Each venue client creates a relay station, which in turn generates its corresponding DOWS client. Entering the name of team venue, we can view the real time simulation updates in our team using the relay stations which relays messages between DOWS application and AG. Note that DOWS application cannot communicate directly with AG.

\section{Conclusion}

This paper have presented the design and implementation of DOWS on AG which supports a collaborative environment for distributed applications. We have shown that the distributed application can be efficiently incorporated into AG environment by proposing a relay station which makes use of event channel and application service APIs in AGTk. Therefore, it provides a way which enables users to integrate applications on a collaborative environment with high quality audio and real-time video interactive interfaces in distributed systems. As a future work, we are working on the integration of computational grid into our system for the performance improvement of computation intensive simulation arising in DOWS.

\section{References}

1. Access Grid http://www.accessgrid.org/

2. Stevens, R., Papka, M.E. and Disz, T.,"Prototyping the workspaces of the future," Internet Computing, IEEE, pp 51-58, July-Aug. 2003.

3. Ho, H. C. and Yang, C. T. and Chang C. C., "Building an E-learning Platform by Access Grid and Data Grid Technologies,"Proceedings of the 2004 IEEE International Conference on e-Technology, pp 121-126 2004.

4. Ernest H. Page and Roger Smith," Introduction to Military Training Simulation: A Guide for Discrete Event Siulationists," Proceedings of the 1998 Winter Simulation Conference, pp 33-40, 1998. 